\title{
Axial loading during MRI induces significant T2 value changes in vertebral endplates-a feasibility study on patients with low back pain
}

Hanna Hebelka ${ }^{1,4^{*}}$ D, Andreia Miron ${ }^{1,4}$, Izabela Kasperska ${ }^{1}$, Helena Brisby ${ }^{2,4}$ and Kerstin Lagerstrand ${ }^{3,4}$

\begin{abstract}
Background: The function of the endplate (EP) is the most important factor influencing nutritional supply to the avascular intervertebral disc (IVD). It is desired to have a non-invasive method to assess functional EP characteristics in vivo. Assessment of functional EP characteristics is important in order to understand its relation to IVD degeneration, which in turn might deepen the understanding of the pathophysiology behind low back pain (LBP). It was hypothesized that, by comparing quantitative MRI of EPs performed with conventional supine MRI (unloaded MRI) with axial loading during MRI (alMRI), dynamical properties of the EP can be displayed. The aim was therefore to investigate the feasibility of axial loading during MRI (alMRI) to instantaneously induce quantitative EP changes.

Methods: T2 mapping of 55 vertebral EPs (L1-S1) in five LBP patients was performed during conventional supine MRI (unloaded MRI) and subsequent alMRI. With T2 mapping, the cartilaginous EP and bony EP cannot be separated; hence, the visualized EP was termed EP zone (EPZ). Each EPZ was segmented at multiple midsagittal views, generating volumetric regions of interest. EPZs demonstrating signal inhomogeneity and/or adjacent Modic changes (MC) were termed abnormal EPZs. EPZ mean T2 values were compared between unloaded MRI and alMRI, and their relationship with abnormal EPZs was determined.

Results: alMRI induced significantly higher $(p=0.01)$ EPZ mean T2 values compared with unloaded MRI. Significantly higher mean T2 values were seen in inferior EPZs compared with superior EPZs, both with unloaded MRI $(35 \%, p<0.001)$ and with alMRI $(26 \%, p=0.04)$. Significant difference between unloaded MRI and alMRI was seen in normal ( $p=0.02)$, but not in abnormal EPZs $(p=0.5 ; n=12)$.

Conclusions: alMRI induces changes in human EPZ characteristics in vivo. The T2 value significantly increased in normal EPZs, with lack of such in abnormal EPZs. Combining T2 mapping with alMRI provides a clinical feasible, non-invasive method with potential to reveal biochemical behavioral patterns, thus adding another dimension of the EPZs characteristics compared with information obtained with solely unloaded MRI.
\end{abstract}

Keywords: Axial loading during MRI (alMRI), IVD, T2 mapping, Endplate

\footnotetext{
* Correspondence: hanna.hebelka@vgregion.se

'Department of Radiology, Sahlgrenska University Hospital, Gothenburg,

Sweden

${ }^{4}$ Institute of Clinical Sciences Sahlgrenska Academy, University of

Gothenburg, Gothenburg, Sweden

Full list of author information is available at the end of the article
} 


\section{Background}

Low back pain (LBP) is an endemic condition with high impact for the individuals affected but also with tremendous socio-economic consequences [1]. LBP is closely associated with both degeneration of the intervertebral disc (IVD) and with vertebral endplate (EP) changes [2-5]. The exact etiological factors for LBP are however still not elucidated and neither are the exact mechanisms initiating the degenerative cascade of the IVD. Further, the lack of a true "gold standard" diagnostic tool that unequivocally points out which spinal structures cause LBP prevents optimal success for used and suggested therapeutic strategies $[6,7]$.

The avascular IVD relies on passage through the EP for nutrient supply and metabolite removal, primarily regulated by diffusion and convection via the vertebral EP $[3,8]$. Dynamic contrast-enhanced MRI (DCE-MRI) has revealed that EPs with signs of degeneration have altered contrast enhancement pattern compared to nondegenerated EPs [9-11]. Hence, structural EP changes, like fissures or sclerosis, may alter its permeability and play a crucial role in the progression of IVD degeneration $[2,3,8,12]$. The exact relationship between compromised EP function and IVD degeneration is not fully understood. To deepen the understanding of the pathophysiology behind IVD degeneration and, in extension, also the understanding of LBP, non-invasive diagnostic tools with ability to in detail characterize early biochemical EP changes are desirable.

Quantitative MRI methods, sensitive for alterations in composition between macromolecules, collagen, and water, reliably reflect changes in biochemical composition and structural integrity in degenerated IVDs [13-16]. In spite of the ability of these methods to reveal early signs of IVD degeneration and thereby potentially find the window when biological targeted therapy might be favorable, quantitative MRI methods are still not used routinely in a clinical setting. Quantitative MRI studies of the EPs are, in contrast to the above discussed IVD studies, limited but have been reported to also reveal subtle deterioration of biochemical EP composition [17-19].

Mechanical loading of the spine has been shown to affect quantitative MRI parameters in studies investigating the IVDs [15, 16, 20-22]; however, similar studies of the EPs are lacking. A recent review regarding the immediate lumbar response to various loading conditions concluded that there is a gap in knowledge on how loading affects spinal imaging [23]. Imaging of the EP in a loaded state versus in an unloaded state might reveal differences in EP characteristics that may reflect altered EP functionality. It was hypothesized that by comparing quantitative MRI of EPs performed with conventional supine MRI (unloaded MRI) with axial loading during MRI (alMRI), dynamical properties of the EP can be displayed. The aim of the present study was to investigate if alMRI instantaneously can induce EP changes, measured with quantitative MRI.

\section{Methods \\ Study participants}

Fifty-five vertebral EPs were investigated in five LBP patients (three female/two male) with mean age 37 years (range 28-49). The patients were recruited, consecutively, among patients included in an ongoing, largescale LBP study. All patients, referred from the spine surgery unit at Sahlgrenska University Hospital, Gothenburg, Sweden, declined any radicular symptoms, and none had undergone previous disc surgery or had any contraindications for performing MRI.

\section{Magnetic resonance imaging}

Each participant was examined with a spinal magnetic resonance imaging (MRI) Siemens Magnetom Aera 1.5T scanner (Erlangen, Germany), consisting of an initial conventional, supine protocol (unloaded MRI) (20 min) followed by an identical repeat supine protocol with the addition of axial loading (alMRI) (20 min). The T2 mapping sequence was included last in the protocols; hence, the T2 mapping with and without axial load was performed for 20 min apart. The alMRI was performed in supine position with a Dynawell compression device (Dynawell diagnostics AB, Las Vegas, NV, USA) with $50 \%$ of the patient's body weight applied to simulate the load in upright position [20]. The protocol constituted of TSE T1- and T2-weighted (W) sequences in the sagittal plane, an axial TSE T2W sequence and a SE T2 mapping sequence in the sagittal plane (Table 1). Calculation of T2 value voxel by voxel was based on multiparametric model.

\section{Measurements}

Since the cartilaginous EP and the bony EP cannot be separated with T2 mapping, the outlined vertebral EP was termed the EP zone (EPZ) (Fig. 1). Since T1 signal overlay on the T2 mapping images improved visibility of the EPZ, segmentation of the EPZ was made on fused sagittal T1 and T2 mapping sequences on the Syngovia (Siemens) image processing software (Fig. 1). To achieve volumetric delineation, the images were reformatted into non-overlapping $10-\mathrm{mm}$ slices. Segmentation was performed on three consecutive $10-\mathrm{mm}$ slices, one midsagittal and two parasagittal. In this fashion, one volumetric segmentation of each EPZ was finally obtained, covering $30 \mathrm{~mm}$ of the median EPZ width in total. Mean T2 values (ms) and standard deviations (SD) of the means (ms) and areas $\left(\mathrm{mm}^{2}\right)$ were recorded for the whole volume. The measurements were repeated in identical fashion on alMRI images. 
Table $1 \mathrm{MRI}$ and alMRI protocol details

\begin{tabular}{|c|c|c|c|c|c|c|}
\hline & NSA (n) & Flip angle & TR (ms) & $\mathrm{TE}(\mathrm{ms})$ & Matrix & FOV $(\mathrm{cm})$ \\
\hline TSE T1 sag & 2 & 150 & 480 & 9 & $320 \times 320$ & $30 \times 30$ \\
\hline TSE T2 sag & 1 & 150 & 3500 & 95 & $384 \times 384$ & $30 \times 30$ \\
\hline TSE T2 ax & 2 & 150 & 5330 & 97 & $320 \times 320$ & $20 \times 20$ \\
\hline SE T2-map & 1 & 150 & 1400 & $11-89(n=8)$ & $256 \times 256$ & $22 \times 22$ \\
\hline
\end{tabular}

Twenty-five IVDs were graded, on T2W sequences, according to Pfirrmann classification [24], with grades 1-2 considered as non-degenerated IVDs while grades 3-5 were considered degenerated.

On the unloaded MR images, each vertebra (L1-S1) was divided in $50 \%$ in cranio-caudal direction to obtain vertebral units directly adjacent to each superior and inferior EP respectively. In each vertebral unit, any existence of Modic changes (MC) were registered and graded as 0 (no changes) and 1 (any $\mathrm{MC}$ type) respectively [5]. In addition, visually observable EPZ changes, like Schmorl's nodules or signal inhomogeneity (i.e., discontinuity of the EPZ, fissures, inhomogeneous signal), in each EPZ were evaluated and graded as 0 (no changes) or 1 (signal inhomogeneity). If signal inhomogeneity or $\mathrm{MC}$ were detected anywhere in the EPZ (30 mm width), it was considered as a positive finding. To term EPZs with either signal inhomogeneity or adjacent to $M C$, the term abnormal EPZs will be used while EPZs without any of these will be termed normal EPZs. It was also registered whether the EPZ was a superior or inferior vertebral EPZ.

All measurements were made by a radiology resident (AM) who also performed intra-observer analysis after 3 months, blinded to the original results. Blinded interobserver analysis was performed by a senior radiologist with over 13 years' experience in the spinal field $(\mathrm{HH})$.

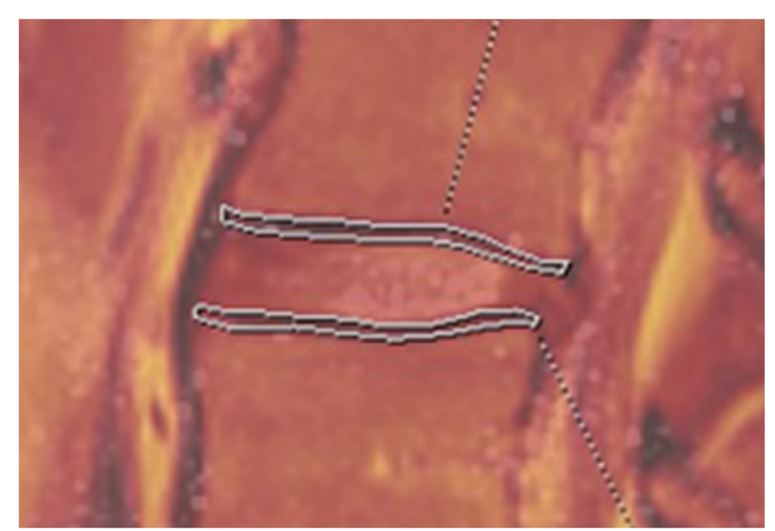

Fig. 1 Illustration of endplate zone (EPZ) segmentation. Illustration of endplate zone (EPZ) segmentation of two EPZs in midsagittal view, on fused T1-weighted and T2 map images respectively

\section{Statistical analysis}

Descriptive data are expressed in terms of mean and standard deviation (SD). A parametric paired $t$ test was used for comparison between differences in dependent T2 values between unloaded MRI and alMRI for the various parameters investigated. Fisher's exact test was used for calculation of the relation between Pfirrmann grade and EP changes. Reliability of quantitative T2 value measurements for both intra- and inter-rater agreement was performed using intraclass correlation coefficient (ICC), model 2, with $95 \%$ confidence intervals. The coefficients were interpreted using Cronbach's alpha [25]. All tests were two-sided, and statistical significance was set as $p<0.05$. Data was analyzed using IBM SPSS Statistics for Windows, Version 22.0. (IBM Corp, Armonk, NY, USA).

\section{Results}

Comparison of all EPZs between unloaded MRI and aIMRI The alMRI induced significantly higher mean T2 values in the EPZs compared with unloaded MRI, with a mean increase of $19 \%(p=0.01)$ (Table 2). When analyzed on disc level, a trend towards reduced differences induced was seen in caudal direction of the lumbar spine (Fig. 2).

\section{Comparison between superior and inferior EPZs}

Significantly higher mean T2 values were seen in inferior EPZs compared with superior EPZs, both with unloaded MRI $(35 \%, p<0.001)$ and with alMRI $(26 \%, p=0.04)$ (Table 2).

\section{EPZ abnormalities and IVD degeneration}

One L5-S1 IVD was sacralized with only a rudimentary IVD. Of the remaining 24 IVDs, none had Pfirrmann grade 5 and only one had grade 1 . In total, eight IVDs

Table 2 T2 values (ms) of endplates zones (EPZ) with unloaded MRI (UMRI) and alMRI

\begin{tabular}{lllllll}
\hline & $N$ & Minimum & Maximum & Mean & SD & $p$ value \\
\hline EPZ uMRI & 55 & 22 & 75 & 36 & 12 & 0.01 \\
EPZ alMRI & 55 & 21 & 80 & 43 & 17 & \\
Inferior EPZ UMRI & 25 & 23 & 75 & 42 & 15 & $<0.001$ \\
Superior EPZ UMRI & 30 & 22 & 49 & 31 & 7 & \\
Inferior EPZ alMRI & 25 & 25 & 80 & 48 & 17 & 0.04 \\
Superior EPZ alMRI & 30 & 21 & 79 & 38 & 16 & \\
\hline
\end{tabular}




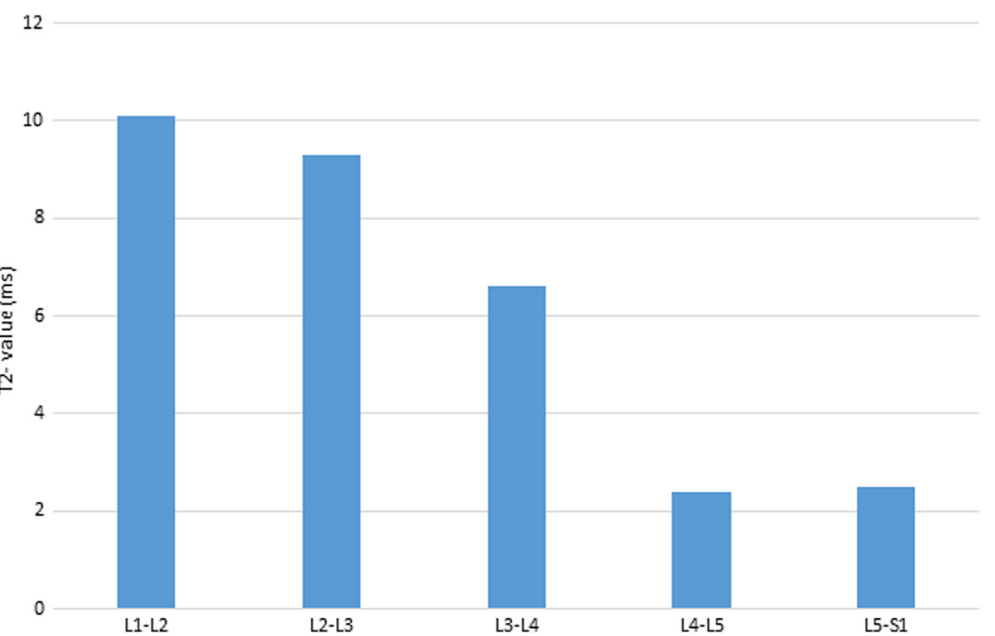

Fig. 2 Mean endplate zone (EPZ) T2 value change for each lumbar level. Difference in endplate zone (EPZ) mean T2 values (ms) between unloaded MRI and alMRI for each lumbar level

were classified as degenerated and 16 non-degenerated. Signal inhomogeneity was detected in $11 \mathrm{EPZs}$, of which six were adjacent to $\mathrm{MCs}$ and nine $(82 \%)$ bordered to degenerated IVDs; MCs (all type 1) were found adjacent to seven EPZs. In total, 12 abnormal EPZs were registered.

\section{Comparison between induced EPZ changes in normal EPZs versus abnormal EPZs}

A significant difference between unloaded MRI and alMRI (from 36 to $43 \mathrm{~ms} ; p=0.03$ ) was found in EPZs without signal inhomogeneity. In EPZs with signal inhomogeneity, no such difference was seen (from 38 to $41 \mathrm{~ms} ; p=0.3)$. In EPZs adjacent to vertebras without MC $(n=48)$, alMRI induced an increased mean T2 value (from 36 to $43 \mathrm{~ms} ; p<0.02$ ). In EPZs adjacent to MCs, no increment in the T2 value was registered (from 42 to $39 \mathrm{~ms} ; p=0.3)$. Significant difference between unloaded MRI and alMRI was seen in normal $(p=0.02)$, but not in abnormal EPZs $(p=0.5 ; n=12)$.

The variation in the T2 value was however large for both normal and abnormal EPZs (Fig. 3). With unloaded MRI, normal EPZs displayed a higher variation $(\mathrm{CV}=36 \%)$ compared with abnormal EPZs $(C V=30 \%)$. With alMRI, the variation in the T2 value increased for normal EPs $(\mathrm{CV}=42 \%)$, however, not for abnormal EPZs $(\mathrm{CV}=29 \%)$.

\section{Reliability measurements}

Reliability measurements of EPZs T2 value showed very good agreement with Crohnbach's alpha 0.86 (95\% CI

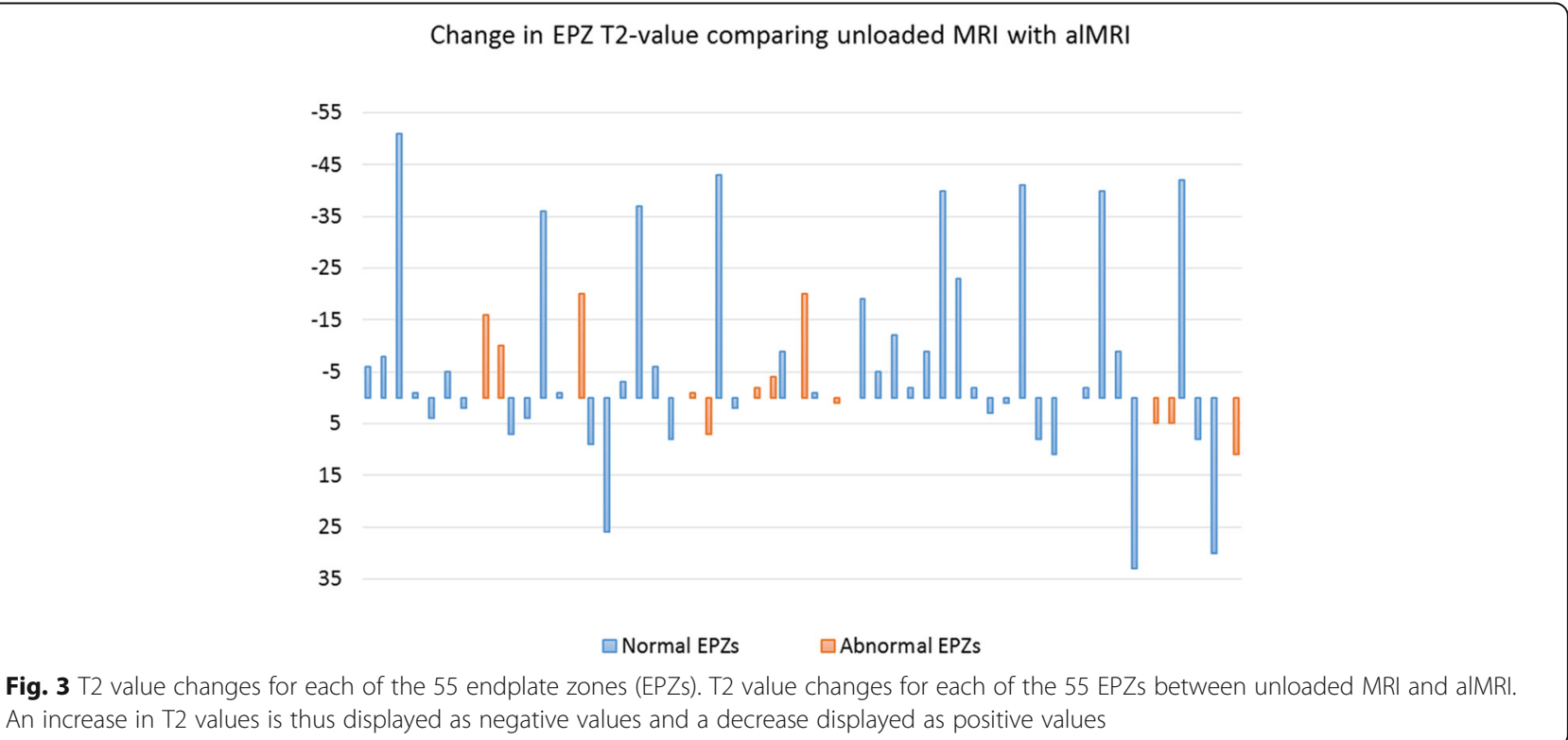


$0.79-0.91)$ for intra-rater measurements and 0.85 (95\% CI 0.78-0.91) for inter-rater agreement respectively.

\section{Discussion}

This feasibility study, comparing T2 values of EPZs obtained by unloaded MRI and alMRI, showed that significantly higher T2 values were induced in the loading state. In abnormal EPZs, no significant induced difference was shown at a group level as opposed to in normal EPZs. Further, the known morphological and functional differences, like for example different stiffness and ability to resist load [26], between superior and inferior EPs were clearly displayed quantitatively.

Spinal load has been shown to affect quantitative MRI parameters of the IVD, which may provide detailed information of the IVD characteristics and its behavioral patterns $[15,16,20-22]$. In this study, it was hypothesized that alMRI would also affect quantitative EP values. The present study seems to confirm this hypothesis by displaying significant induced changes in normal EPZs, however, not in abnormal EPZs. Hence, combining T2 mapping with alMRI adds another dimension of the EPZ characteristics compared with information obtained with solely unloaded MRI and may, thus, provide a clinical feasible, non-invasive method with potential to reveal biochemical behavioral patterns of the EPZs.

The cartilaginous EP consists of approximately 50-60\% water [18], with a high degree of transport in the wellhydrated matrix. With degeneration, the permeability of the EP decreases due to occlusion of the vascular buds and increased ratio of calcification and collagen content $[18,27,28]$ which restrict the nutritional transport over the EP $[8,9,27]$. EP damage can however paradoxically also result in increased permeability with loss of essential nutrients and enzymes [9]. For example, Rajesekaran et al. performed serial post contrast-enhanced MRI sequences in 54 individuals and reported either enhanced diffusion or delayed such as in EPs with degenerative signs (fissures, Schmorls, etc.), showing that type of EP injury results in different functional behavior $[8,9,27]$. In general, higher $\mathrm{T} 2$ values are interpreted as high hydration. However, this is a simplified model since T2 values reflect not only the composition between proteoglycans, collagen, and water but also depend on tissue anisotropy, i.e., matrix organization $[14,15,21,22,29,30]$. Why certain EPZs respond to load in different ways are obviously complex and probably multifactorial. The differences between abnormal and normal EPZs seen in the current study show, however, that the EPZs display different characteristics, and the lack of response from alMRI in abnormal EPZs may be a reflection of impaired integrity.

Despite the fact that alMRI induced an increase in the EPZ T2 value on a group level, on an individual EPZlevel, a large variation in the induced changes were seen, from $187 \%$ increase to $55 \%$ decrease (Fig. 3). In abnormal EPZs, the range of induced changes was not as pronounced ( 89 and $26 \%$ respectively). This wide range of T2 values may in fact reflect large differences in the characteristics between individual $E P Z s$, and the smaller range of values in abnormal EPZs may reflect a lower dynamical variation due to impaired EP function. Future studies are warranted to strengthen these results and to investigate the pathophysiological mechanisms behind this divergent behavior between normal and abnormal EPZs.

Mechanical stimuli can exert both anabolic and catabolic effects on the spine $[17,29-31]$. The effect that alMRI exert on each EP is not known, and it cannot be excluded that this instantaneous load do not impair EP transport at some levels. However, since alMRI aims to simulate the load under upright position, alMRI have been assumed to exert load within a physiological beneficial range [32]. Other potential factors that might influence the various alMRI-induced EPZ responses are the level of the EPZ in the lumbar spine, the lordosis and IVD angle induced, and the status of the EPZ and IVD. Recently, alMRI was shown to induce regional alterations in IVD T2 values depending on IVD level [20]. The present feasibility study was not powered to investigate such relations. Induced differences however seemed to decline in caudal direction of the lumbar spine, which speculatively might be explained by degenerative changes being more common caudally. Out of 16 degenerated IVDs, 12 were localized at L4-S1 level as were nine of the 12 abnormal EPZs.

The absolute $\mathrm{T} 2$ values obtained in the present study were slightly lower (22-74 ms unloaded MRI) than previously reported by Delucca et al. (60-100 ms) [28], a discrepancy likely due to either the previous cadaver study design or that outlining of only the cartilaginous EP was not possible in the current in vivo study. The significant difference in absolute T2 values between superior and inferior EPZs likely reflects what has been shown in biomechanical in vitro studies and animal in vivo with the inferior EP being stiffer, resist load better and with different transport kinetics compared with the superior EP [26, 30]. This phenomenon is reflected in clinical situations where for example superior EPs being more prone to burst fractures [33]. Also human in vivo studies show that contrast enhancement differ between superior and inferior vertebral EPs [10, 11]. To our knowledge, this is the first human in vivo study displaying quantitative differences, which may reflect morphological differences, between the superior and inferior EPZs. Thus, these results constitute ground data for future studies.

Data from a single time point cannot fully characterize the complex kinetics occurring in the EP and why 
comparing induced changes between unloaded MRI and alMRI provides another dimension, indicating dynamic EP characteristics. Such has been reported promising in the IVD [20], and this study shows that alMRI is feasible to induce changes also in the less hydrated EP. Despite large differences at individual levels, significant differences were found at group level and in addition with a strong test-retest repeatability, making the results of this feasibility study worth studying in large-scale studies.

The distinct responses induced with alMRI makes the combination of quantitative MRI and alMRI a promising method to assess behavioral EP characteristics clinically. In addition, alMRI offers a method to image the spine in a position inducing concordant pain in LBP patients [34]. To elucidate if the differences in EPZ characteristics between unloaded MRI and alMRI represent altered diffusion/perfusion, future studies are encouraged to include diffusionweighted sequences. If this method can reveal also early signs of impaired EP integrity, before manifest IVD degeneration appears, it would provide important information that ultimately may be used for targeted therapy. To evaluate clinical application of the method, work has been initiated to elucidate if this method can depict pain predictors by comparing if the induced EP T2 value changes vary between a large-scale LBP cohort and healthy controls.

The small sample size is a limitation. In a previous alMRI study that investigated IVD changes [20] (50 IVDs), significant changes were reported, why the current sample size was considered appropriate to evaluate if it is feasible to induce EPZ T2 value changes with alMRI. However, considering the limited number $(n=12)$ of abnormal EPZs, it cannot be excluded that this study is underpowered for evaluation of behavioral pattern regarding abnormal EPZs. The so-called "magic angle effect," appearing at approximately $55^{\circ}$ from the main magnetic field [18], must be considered. However, the effect in the current study is regarded as non-existing at L1-L4 and only minor at L5-S1 since none of the EPZs at level L1-L4 were imaged between $50^{\circ}$ and $60^{\circ}$ with unloaded MRI and only one L5 EPZ was imaged at $58^{\circ}$ and four S1 EPZs imaged at $53^{\circ}-54^{\circ}$. With alMRI only, three EPZs were imaged between $50^{\circ}$ and $60^{\circ}$.

Contribution of minor signal from adjacent tissue, for example the IVD, cannot be completely excluded due to the image resolution obtained with T2 mapping. However, this potential partial volume effect ought to be minor on the whole segmented EPZ volume and in addition negligible regarding induced EPZ changes since such effect ought to be similar on unloaded MRI and alMRI.

\section{Conclusion}

This study shows, for the first time, that alMRI induce changes in human EPZs characteristics in vivo. However, when analyzed on group level, the T2 value significantly increased in normal EPZs, with lack of such in abnormal EPZs. Combining T2 mapping with alMRI provides a clinical feasible, non-invasive method with potential to reveal biochemical behavioral EP patterns, thus adding another dimension of the EPs characteristics compared with information obtained with solely unloaded MRI. Thus, this study opens up an area for future research since imaging during loading conditions might increase the diagnostic specificity, providing new biomarkers, within spine imaging.

\section{Abbreviations}

alMRI: Axial loading during MRI; EP: Endplate; EPZ: Endplate zone; ICC: Intraclass correlation coefficient; IVD: Intervertebral disc; LBP: Low back pain; MC: Modic changes; MRI: Magnetic resonance imaging

\section{Acknowledgements}

The authors thank Christer Johansson, OrigoVerus AB, Gothenburg, Sweden, for statistical assistance.

\section{Funding}

The authors acknowledge the financial support from C4I Center, Sahlgrenska University Hospital and from Konrad and Helfrid Johansson's Foundation.

Financial support has been used to cover expenses for examinations and for manuscript writing. The study sponsors had no influence on the analysis and interpretation of data; nor in the writing of the report.

\section{Availability of data and materials}

The datasets used and/or analyzed during the current study are available from the corresponding author on reasonable request.

\section{Authors' contributions}

$\mathrm{KL}$ and $\mathrm{HH}$ are responsible for the study design. $\mathrm{AM}$ and $\mathrm{HH}$ performed the measurement of $\mathrm{T} 2$ values. $\mathrm{KL}$ performed part of the statistical analysis. $\mathrm{KL}$, $\mathrm{HH}, \mathrm{AM}$, and $\mathrm{HB}$ analyzed and interpreted the patient data. $\mathrm{HH}$ and $\mathrm{KL}$ were major contributors in writing the manuscript. IK performed the MRI examinations and was responsible for the administration regarding ethical considerations of the study. All authors read and approved the final manuscript.

\section{Ethics approval and consent to participate}

All procedures performed in studies involving human participants were in accordance with the Regional Ethics Review Board at the region of Västra Götaland and with the 1964 Helsinki declaration and its later amendments or comparable ethical standards. Oral and written informed consent was obtained from all individual participants included in the study.

\section{Consent for publication \\ Not applicable.}

\section{Competing interests}

All authors declare that they have no competing interests.

\section{Publisher's Note}

Springer Nature remains neutral with regard to jurisdictional claims in published maps and institutional affiliations.

\section{Author details}

'Department of Radiology, Sahlgrenska University Hospital, Gothenburg, Sweden. ${ }^{2}$ Department of Orthopaedics, Sahlgrenska University Hospital, Gothenburg, Sweden. ${ }^{3}$ Department of Medical Physics and Techniques, Sahlgrenska University Hospital, Gothenburg, Sweden. ${ }^{4}$ Institute of Clinical 
Sciences Sahlgrenska Academy, University of Gothenburg, Gothenburg, Sweden

\section{Received: 4 December 2017 Accepted: 19 January 2018}

Published online: 30 January 2018

\section{References}

1. Katz JN. Lumbar disc disorders and low-back pain: socioeconomic factors and consequences. J Bone Joint Surg Am. 2006;88:21-4.

2. Adams MA, Freeman BJ, Morrison HP, Nelson IW, Dolan P. Mechanical initiation of intervertebral disc degeneration. Spine. 2000;25:1625-36.

3. Urban JP, Smith S, Fairbank JC. Nutrition of the intervertebral disc. Spine. 2004;29:2700-9.

4. Luoma K, Riihimäki H, Luukkonen R, Raininko R, Viikari-Juntura $E$, Lamminen A. Low back pain in relation to lumbar disc degeneration. Spine. 2000;25:487-92

5. Modic M, Steinberg P, Ross J, Masaryk T, Carter J. Degenerative disk disease: assessment of changes in vertebral body marrow with MR imaging. Radiology. 1988;166:193-9.

6. Endean A, Palmer KT, Coggon D. Potential of magnetic resonance imaging findings to refine case definition for mechanical low back pain in epidemiological studies: a systematic review. Spine (Phila Pa 1976). 2011;36:160-9.

7. Chou R, Fu R, Carrino JA, Deyo RA. Imaging strategies for low-back pain: systematic review and meta-analysis. Lancet. 2009;373:463-72.

8. Van Der Werf M, Lezuo P, Maissen O, Van Donkelaar CC, Ito K. Inhibition of vertebral endplate perfusion results in decreased intervertebral disc intranuclear diffusive transport. J Anat. 2007;211:769-74.

9. Rajasekaran S, Babu JN, Arun R, BRW A, Shetty AP, Murugan S. ISSLS prize winner: a study of diffusion in human lumbar discs: a serial magnetic resonance imaging study documenting the influence of the endplate on diffusion in normal and degenerate discs. Spine. 2004;29:2654-67.

10. Muftuler LT, Jarman JP, Hon JY, Gardner VO, Maiman DJ, Arpinar VE. Association between intervertebral disc degeneration and endplate perfusion studied by DCE-MRI. Eur Spine J. 2015;24:679-85.

11. Arpinar VE, Rand SD, Klein AP, Maiman DJ, Muftuler LT. Changes in perfusion and diffusion in the endplate regions of degenerating intervertebral discs: a DCE-MRI study. Eur Spine J. 2015;24:2458-67.

12. Benneker LM, Heini PF, Alini M, Anderson SE, Ito K. 2004 young investigator award winner: vertebral endplate marrow contact channel occlusions and intervertebral disc degeneration. Spine. 2005;30:167-73.

13. Pandit P, Talbott JF, Pedoia V, Dillon W, Majumdar S. T1 $\rho$ and T2-based characterization of regional variations in intervertebral discs to detect early degenerative changes. J Orthop Res. 2016:34:1373-81.

14. Antoniou J, Mwale F, Demers CN, Beaudoin G, Goswami T, Aebi M, Alini M. Quantitative magnetic resonance imaging of enzymatically induced degradation of the nucleus pulposus of intervertebral discs. Spine. 2006:31:1547-54.

15. Mwale F, latridis JC, Antoniou J. Quantitative MRI as a diagnostic tool of intervertebral disc matrix composition and integrity. Eur Spine J. 2008; 17(Suppl 4):432-40.

16. Chiu EJ, Newitt DC, Segal MR, Hu SS, Lotz JC, Majumdar S. Magnetic resonance imaging measurement of relaxation and water diffusion in the human lumbar intervertebral disc under compression in vitro. Spine (Phila Pa 1976). 2001;26:E437-44.

17. Chen C, Jia Z, Han Z, Gu T, Li W, Li H, Tang Y, Wu J, Wang D, He Q. Quantitative $\mathrm{T} 2$ relaxation time and magnetic transfer ratio predict endplate biochemical content of intervertebral disc degeneration in a canine model. BMC Musculoskelet Disord. 2015;16:157.

18. Fields AJ, Han M, Krug R, Lotz JC. Cartilaginous end plates: quantitative MR imaging with very short echo times-orientation dependence and correlation with biochemical composition. Radiology. 2014;274:482-9.

19. Bae WC, Biswas R, Chen K, Chang EY, Chung CB. UTE MRI of the osteochondral junction. Current Radiology Reports. 2014;2:35.

20. Nilsson M, Lagerstrand K, Kasperska I, Brisby H, Hebelka H. Axial loading during MRI influences T2-mapping values of lumbar discs: a feasibility study on patients with low back pain. Eur Spine J. 2016;25:2856-63.

21. Perie D, latridis J, Demers C, Goswami T, Beaudoin G, Mwale F, Antoniou J. Assessment of compressive modulus, hydraulic permeability and matrix content of trypsin-treated nucleus pulposus using quantitative MRI. J Biomech. 2006;39:1392-400.
22. Mwale F, Demers CN, Michalek AJ, Beaudoin G, Goswami T, Beckman L, latridis JC, Antoniou J. Evaluation of quantitative magnetic resonance imaging, biochemical and mechanical properties of trypsin-treated intervertebral discs under physiological compression loading. J Magn Reson Imaging. 2008;27:563-73.

23. Abdollah $\mathrm{V}$ : The effects of axial loading on the disc and motion segments relative to disc degeneration and pain using novel MRI biomarkers. University of Alberta, Faculty of Rehabilitation Medicine; 2017.

24. Pfirrmann CW, Metzdorf A, Zanetti M, Hodler J, Boos N. Magnetic resonance classification of lumbar intervertebral disc degeneration. Spine (Phila Pa 1976). 2001:26:1873-8

25. Landis JR, Koch GG. The measurement of observer agreement for categorical data. Biometrics. 1977;33:159-74.

26. Grant JP, Oxland TR, Dvorak MF. Mapping the structural properties of the lumbosacral vertebral endplates. Spine. 2001;26:889-96.

27. Roberts S, Urban JP, Evans H, Eisenstein SM. Transport properties of the human cartilage endplate in relation to its composition and calcification. Spine. 1996;21:415-20.

28. DeLucca JF, Peloquin JM, Smith LJ, Wright AC, Vresilovic EJ, Elliott DM. MRI quantification of human spine cartilage endplate geometry: comparison with age, degeneration, level, and disc geometry. J Orthop Res. 2016:34:1410-7.

29. Gullbrand SE, Ashinsky BG, Martin JT, Pickup S, Smith LJ, Mauck RL, Smith HE. Correlations between quantitative T2 and T1 $\rho$ MRI, mechanical properties and biochemical composition in a rabbit lumbar intervertebral disc degeneration model. J Orthop Res. 2016:34:1382-8.

30. Gullbrand SE, Peterson J, Mastropolo R, Roberts TT, Lawrence JP, Glennon JC, DiRisio DJ, Ledet EH. Low rate loading-induced convection enhances net transport into the intervertebral disc in vivo. Spine J. 2015;15:1028-33.

31. Arun R, Freeman BJ, Scammell BE, McNally DS, Cox E, Gowland P. 2009 ISSLS prize winner: what influence does sustained mechanical load have on diffusion in the human intervertebral disc?: an in vivo study using serial postcontrast magnetic resonance imaging. Spine. 2009;34:2324-37.

32. Wilke HJ, Neef $P$, Caimi M, Hoogland T, Claes LE. New in vivo measurements of pressures in the intervertebral disc in daily life. Spine. 1999;24:755-62.

33. Magerl F, Aebi M, Gertzbein S, Harms J, Nazarian S. A comprehensive classification of thoracic and lumbar injuries. Eur Spine J. 1994;3:184-201.

34. Hebelka H, Brisby $H$, Hansson T. Comparison between pain at discography and morphological disc changes at axial loaded MRI in patients with low back pain. Eur Spine J. 2014;23:2075-82.

\section{Submit your next manuscript to BioMed Central and we will help you at every step:}

- We accept pre-submission inquiries

- Our selector tool helps you to find the most relevant journal

- We provide round the clock customer support

- Convenient online submission

- Thorough peer review

- Inclusion in PubMed and all major indexing services

- Maximum visibility for your research

Submit your manuscript at www.biomedcentral.com/submit
Biomed Central 